\title{
Lernen-aprender: una aproximación contrastiva dentro del campo semántico Kognition en torno a diversas peculiaridades semánticas y sintácticas ${ }^{1}$
}

\author{
Paloma SÁNCHEZ HERNÁNDEZ \\ Universidad Complutense de Madrid \\ palomash@filol.ucm.es
}

Recibido: 27 de noviembre de 2011

Aceptado: 8 de febrero de 2012

\begin{abstract}
RESUMEN
En este estudio se presenta un análisis contrastivo en torno al campo semántico Kognition. Dentro de este campo se trabajará con seis verbos, tres en alemán y tres en español: lernen, üben, studieren, aprender, practicar, estudiar. Para realizar el análisis contrastivo de los lexemas se incluirá diversa información de tipo semántico y sintáctico procedente de diversos diccionarios. Los ejemplos de uso tomados del corpus COSMAS II del IdS, para el alemán, y CREA, para el español, servirán para comparar la información de los diccionarios con el uso real de los lexemas. El objetivo es intentar mostrar las similitudes y diferencias en el funcionamiento de las dos lenguas, así como la relevancia que la información lexicográfica tiene en este tipo de estudios.
\end{abstract}

Palabras clave: Campo semántico, sintaxis, lexicología, lexicografía.

\section{Lernen-aprender: A Contrastive Approach in the Semantic Field of Kognition around some Semantic and Syntactic Peculiarities}

\begin{abstract}
This research paper deals with the semantic field of Kognition from a contrastive point of view. Within this semantic field, we shall analyze six verbal lexemes, three in German and three in Spanish, as there are: lernen, üben, studieren, aprender, practicar, estudiar. A contrastive analysis of these lexemes will be established according to their semantic and syntactic characteristics which have been consulted in monolingual and bilingual dictionaries. Besides, authentic examples of use from COSMAS II in German and CREA in Spanish will complete the study of divergences between normalized language and the real use of these lexemes. The paper aims at showing the similarities and differences in both languages, as to semantic and syntactic functions, as well as the relevance of the lexicographic information for this type of studies.
\end{abstract}

Keywords: Semantic Field, Syntax, Lexicology, Lexicography.

\footnotetext{
${ }^{1}$ Este artículo se enmarca dentro de los estudios correspondientes al proyecto de investigación: "Estudios para la elaboración de un diccionario conceptual de lexemas verbales del alemán y del español” (Xunta de Galicia: 10PXIB 204188 PR).
} 
SUMARIO: 1. Introducción. 2. Campo semántico en alemán. 3. Campo semántico en español. 4. Análisis contrastivo alemán-español-alemán. 5. Conclusión.

\section{Introducción}

Este estudio está elaborado sobre la base de un seminario celebrado en la Universidad de Santiago de Compostela desde el 23 al 26 de septiembre de 2011. En este seminario se discutieron una serie de ámbitos lexicológicos y lexicográficos en el marco del proyecto DICONALE. En este trabajo se intentarán reflejar algunos aspectos semánticos y sintácticos que fueron objeto de discusión en el seminario.

Hartmann (1991: 2854) alude al "análisis contrastivo" como disciplina joven, en comparación con la tradición que muestran los estudios comparativos en lingüística. La relevancia de estos análisis para la lexicografía bilingüe no ha sido investigado antes de un modo sistemático, a excepción, quizá, en relación con el término "equivalencia" usado en traducción ${ }^{2}$.

La descripción lexicográfica propuesta se asienta sobre las bases de la lexicografía bilingüe desde un punto de vista onomasiológico, (Reichmann 1990: 1063) con indicación de información paradigmática (Hausmann 1991: 2795) y sintagmática (Cowie 1989: 590; Model 2006: 250; Cop 1991: 2776), que sirva al usuario para la producción de textos a un nivel avanzado.

El campo semántico analizado en este proyecto es Kognition ${ }^{3}$. De este campo, se ha elegido un subcampo: das Lernen. El corpus está formado por tres verbos en alemán y tres verbos en español: üben, studieren, lernen, practicar, estudiar y aprender. La selección del corpus se ha hecho mediante diccionarios onomasioló-

\footnotetext{
${ }^{2}$ Hartmann opina, además, que no hay una relación directa o mutua entre análisis contrastivos y lexicografía bilingüe. La descripción descriptiva de un conjunto de fenómenos desde un par concreto de lenguas puede dar lugar a una codificación de conceptos que representan estos fenómenos en el diccionario bilingüe. Sin embargo, es cuestionable si este trabajo contrastivo afecta siempre materialmente a la práctica lexicográfica y, aparte de esto, puede ser el diccionario bilingüe el que ofrezca al lingüista los datos o la verificación de los mismos. En cuanto a la lexicología contrastiva, Hartmann es de la opinión que el análisis componencial podría ser una solución para lograr la aplicación de los estudios contrastivos a la lexicografía bilingüe: "just a phonological units may be broken down into distinct phonetic features, lexemes are held to be capable of analysis into senses as 'complexes of semantic features'. Thus, a contrastive analysis of the different senses of a word like contrast would not only be regarded as revealing their equivalent(s) in other languages, but also seen as improving the arrangement of the information within the dictionary entry itself' (HARTMANN 1991: 2856-2857). No obstante, Hartmann alude también a la dificultad real para implementar tal relación por los problemas referidos a las interferencias en la adquisición de lenguas, entre otros.

${ }^{3}$ Kognition: "Gesamtheit aller Prozesse, die mit dem Wahrnehmen und Erkennen zusammenhängen”. (DUDEN 2007 s/v: Kognition). Según Coseriu, un campo semántico es: "un paradigma léxico que se origina por la distribución de un continuo de contenido léxico en diferentes unidades, dadas en la lengua como palabras, que están recíprocamente en oposición inmediata mediante rasgos distintivos de contenido simple" (COSERIU 1967: 294).
} 
gicos, así como con la ayuda de diccionarios de sinónimos ${ }^{4}$.También se han tenido en cuenta algunos trabajos anteriores sobre el mismo campo semántico ${ }^{5}$. Aparte de éste, no se conocen otros trabajos en el ámbito de la lexicografía bilingüe contrastiva alemán-español dentro del subcampo das Lernen.

En este trabajo se ha intentado establecer un estudio de algunas características semánticas y sintácticas de cada uno de los elementos del campo. Además de una perífrasis del significado, en cada una de las acepciones de cada lexema se ofrecerán las características sintácticas y la valencia semántica de los elementos. En cuanto a las características sintácticas, se añaden dos tipos de información: la estructura sintáctica, es decir, el funcionamiento real del verbo en la oración, y el tipo de actantes, esto es, la función de cada elemento, la cual, si corresponde a un actante facultativo, aparece entre paréntesis ${ }^{6}$. La valencia semántica está referida a los rasgos semánticos ${ }^{7}$ más relevantes de cada uno de los elementos de la oración. Un aspecto interesante será comparar esta valencia semántica en ambas lenguas, de manera que se pueda analizar cuándo ésta coincide y cuándo no. En algunos casos, puede sumarse al análisis algún tipo de información sobre el registro lingüístico, cuando sea relevante. Se añade, además, un ejemplo de uso procedente del corpus del IdS COSMAS (https://cosmas2.ids-mannheim.de), en alemán, y de CREA y el corpus del español de Mark Davies, en español. La inclusión de ejemplos reales de corpus sirve para poner de manifiesto la estructura semántica y sintáctica que se postula en cada caso. Esta información se ha tomado de diversos diccionarios sintagmáticos como Engel, U. / Schumacher, H. (1978), Helbig, G. / Schenkel, W. (1980) y Schumacher, H. / Kubczak, J. / Schmidt, R. / de Ruiter, V. (2004). El estudio se realizará en primer lugar con los lexemas en alemán, en un segundo momento, con los lexemas en español y, por último, se realizará el estudio contrastivo.

\section{Campo semántico en alemán}

\section{1. üben}

Una de las cuestiones que surgieron al analizar el lexema üben fue si este lexema pertenecía realmente al campo semántico objeto del estudio. Según su significado, se han incluido sólo aquellas acepciones de üben donde éste pertenece directamente a este campo semántico, es decir, donde su sentido está relacionado directamente con el subcampo aprender o con el campo Kognition. El lexema üben posee dos acepciones (cfr. Fig. 1 y 2):

\footnotetext{
${ }^{4}$ Me refiero en este punto a WeHrLe-EgGers (1961), DornSEIFF (1965) RADSZUWEIT e.a. (1992), CASARES (1994) y CORRIPIO (1990).

${ }^{5}$ Véase SÁNCHEZ HERNÁNDEZ (2010: 261-283).

${ }^{6} \mathrm{He}$ de decir que, en este estudio, se ha seguido la corriente sintáctica de ENGEL (1978), ENGEL (2004) y la Dependenzgrammatik, aunque también se ha intentado simplificar al máximo la nomenclatura.

${ }^{7}$ Utilizo rasgos semánticos o semas y lexemas en sentido de la semántica estructural: "Los rasgos semánticos distintivos (mínimos) en el análisis del contenido pueden llamarse semas" (GECKELER 1994: 233). "[...] Toda unidad dada en la lengua como palabra simple es, desde el punto de vista del contenido, un lexema" (COSERIU 1967: 294).
} 


\begin{tabular}{|c|c|c|c|c|c|c|}
\hline & Significado & $\begin{array}{l}\text { Tipo de } \\
\text { actantes }\end{array}$ & \multicolumn{2}{|c|}{$\begin{array}{l}\text { Estructura } \\
\text { sintáctica }\end{array}$} & $\begin{array}{l}\text { Valencia } \\
\text { semántica }\end{array}$ & \\
\hline & & & & & sub & akk \\
\hline üben1 & $\begin{array}{l}\text { etwas immer } \\
\text { wieder tun, } \\
\text { um es zu } \\
\text { erlernen und } \\
\text { dann besser zu } \\
\text { beherrschen } \\
\text { (DWDS). }\end{array}$ & $\begin{array}{l}\text { sub } \\
(\mathrm{akk})\end{array}$ & $\begin{array}{l}\text { jmd [ } \\
\text { (etwa }\end{array}$ & $\begin{array}{l}\text { übt } \\
\text { akk) }\end{array}$ & $\begin{array}{l}+ \text { anim }] \\
{[+ \text { Mensch }]} \\
{[+ \text { Tiere }]}\end{array}$ & $\begin{array}{l}\text { Facultativo } \\
{[- \text { mat }]} \\
{[+ \text { Zustand }]} \\
{[+ \text { Handlung }]}\end{array}$ \\
\hline \multicolumn{4}{|c|}{$\begin{array}{l}\text { COSMAS II: BRZ06/JUL.05492 } \\
\text { Braunschweiger Zeitung, 11.07.2006; } \\
\text { "Grammatik und Vokabeln üben gehörten } \\
\text { sowieso dazu. 'Ohne die ist eine Sprache, } \\
\text { als wolle man ein Haus ohne Fundamente } \\
\text { bauen'. Faszinierend seien die } \\
\text { Präsentationen, die alle Schüler in der } 11 . \\
\text { Klasse vorbereiten müssen". }\end{array}$} & \multicolumn{2}{|c|}{$\begin{array}{l}\text { Posible } \\
\text { correspondencia } \\
\text { en español: } \\
\text { practicar }\end{array}$} & \\
\hline
\end{tabular}

Figura 1

\begin{tabular}{|c|c|c|c|c|c|c|}
\hline & Significado & $\begin{array}{l}\text { Tipo de } \\
\text { actantes }\end{array}$ & \multicolumn{2}{|c|}{$\begin{array}{l}\text { Estructura } \\
\text { sintáctica }\end{array}$} & $\begin{array}{l}\text { Valencia } \\
\text { semántica }\end{array}$ & \\
\hline & & & & & sub & prp \\
\hline üben2 & $\begin{array}{l}\text { versuchen, } \\
\text { etwas } \\
\text { anzuwenden, } \\
\text { etw. oft, viel } \\
\text { üben oder tun } \\
\text { (DWDS). }\end{array}$ & sub prp & $\begin{array}{l}\text { jmd [st } \\
\text { sich in } \\
{[\text { in }+ \text { Da }}\end{array}$ & $\begin{array}{l}\text { b] übt } \\
\text { etwas } \\
\text { t] (geh.) }\end{array}$ & $\begin{array}{l}+ \text { anim }] \\
{[+ \text { Mensch }]} \\
{[+ \text { Tier }]}\end{array}$ & $\begin{array}{l}\text { in+Dativ } \\
{[- \text { mat }]} \\
\text { [+Zustand }] \\
\text { [+Handlung }]\end{array}$ \\
\hline \multicolumn{4}{|c|}{$\begin{array}{l}\text { COSMAS II:HMP10/JAN.02946 } \\
\text { Hamburger Morgenpost, } 31.01 .2010 \text {; } \\
\text { "Wichtig ist, sich im Smalltalk zu üben und } \\
\text { kleine positive Erfahrungen zu machen. } \\
\text { Das kann eine Plauderei im Wartezimmer } \\
\text { sein oder in der Schlange an der Käsetheke. } \\
\text { Die kleinen Alltagsflirts senken auf Dauer } \\
\text { die Hemmschwelle". }\end{array}$} & \multicolumn{2}{|c|}{$\begin{array}{l}\text { Posible correspon- } \\
\text { dencia en español: } \\
\text { practicar }\end{array}$} & \\
\hline
\end{tabular}

Figura 2

En este sentido, hay que destacar el uso de üben 2 en la lengua culta, respecto de übenl. 


\section{2. studieren}

El verbo studieren posee tres acepciones en alemán (cfr. Fig. 3, 4, 5):

\begin{tabular}{|c|c|c|c|c|c|}
\hline & Significado & $\begin{array}{l}\text { Tipo de } \\
\text { actantes }\end{array}$ & $\begin{array}{l}\text { Estructura } \\
\text { sintáctica }\end{array}$ & $\begin{array}{l}\text { Valencia } \\
\text { semántica }\end{array}$ & \\
\hline & & & & sub & akk \\
\hline studieren 1 & $\begin{array}{l}\text { Eine } \\
\text { Hochschule } \\
\text { besuchen; an } \\
\text { einer } \\
\text { Hochschule } \\
\text { Wissen, } \\
\text { Kenntnisse } \\
\text { auf einem } \\
\text { bestimmten } \\
\text { Fachgebiet } \\
\text { erwerben } \\
\text { (DUW). }\end{array}$ & sub (akk) & $\begin{array}{l}\text { jmd [sub] } \\
\text { studiert } \\
\text { (etwas) } \\
\text { (akk) }\end{array}$ & $\begin{array}{l}+ \text { anim }] \\
{[+ \text { Mensch }]} \\
{[+ \text { Erwachsen }]}\end{array}$ & $\begin{array}{l}\text { facultativo } \\
\text { [-anim] } \\
\text { [-mat] } \\
\text { [+Fachgebiet] } \\
\text { [+Ideen]; } \\
\text { sin artículo: sie } \\
\text { studiert Chemie }\end{array}$ \\
\hline \multicolumn{3}{|c|}{$\begin{array}{l}\text { COSMAS II: Ejemplo: COSMAS II: } \\
\text { HMP10/APR.00046 Hamburger } \\
\text { Morgenpost, 01.04.2010, p. 20; “13000 } \\
\text { junge Leute studieren heute an der HAW }\end{array}$} & \multicolumn{2}{|c|}{$\begin{array}{l}\text { Posible corres- } \\
\text { pondencia en } \\
\text { español: hacer } \\
\text { una carrera uni- } \\
\text { versitaria, estu- } \\
\text { diar en la univer- } \\
\text { sidad }\end{array}$} & \\
\hline
\end{tabular}

Figura 3

\begin{tabular}{|c|c|c|c|c|c|c|}
\hline & Significado & $\begin{array}{l}\text { Tipo de } \\
\text { actantes }\end{array}$ & \multicolumn{2}{|c|}{$\begin{array}{l}\text { Estructura } \\
\text { sintáctica }\end{array}$} & $\begin{array}{l}\text { Valencia } \\
\text { semántica }\end{array}$ & \\
\hline & & & \multicolumn{2}{|c|}{$\begin{array}{l} \\
\text { jmd [sub] } \\
\text { studiert } \\
\text { etwas [akk] }\end{array}$} & sub & akk \\
\hline studieren2 & $\begin{array}{l}\text { etwas } \\
\text { wissenschaft- } \\
\text { lich, genau } \\
\text { untersuchen } \\
\text { (DUW). }\end{array}$ & sub akk & $\begin{array}{l}\text { jmd } \\
\text { stud } \\
\text { etwa }\end{array}$ & $\begin{array}{l}\text { ub] } \\
\mathrm{rt} \\
{[\mathrm{akk}]}\end{array}$ & $\begin{array}{l}{[+ \text { anim }]} \\
{[+ \text { Mensch }} \\
]\end{array}$ & $\begin{array}{l}{[\text {-anim }]} \\
{[+ \text { anim }]} \\
\text { [+mat }] \text { (Akten) } \\
\text { [+Handlungen } \\
\text { ] [+Zustände } \\
\text { [+Ideen] } \\
\text { [+Mensch }\end{array}$ \\
\hline \multicolumn{4}{|c|}{$\begin{array}{l}\text { COSMASII: IINON07/JAN.11219 } \\
\text { Niederösterreichische Nachrichten, 23.01.2007, p. } \\
\text { 25; "Weniger Delikte - weniger Erfolge } \\
\text { WAIDHOFEN / Weniger zu tun hatten die } \\
\text { Beamten im Vergleich zum Jahr 2005, wenn man } \\
\text { die aktuelle Kriminalstatistik studiert". }\end{array}$} & \multicolumn{2}{|c|}{$\begin{array}{l}\text { Posible corres- } \\
\text { pondencia en } \\
\text { español: estudiar }\end{array}$} & \\
\hline
\end{tabular}

Figura 4 


\begin{tabular}{|c|c|c|c|c|c|c|}
\hline & Significado & $\begin{array}{l}\text { Tipo de } \\
\text { actantes }\end{array}$ & \multicolumn{2}{|c|}{$\begin{array}{l}\text { Estructura } \\
\text { sintáctica }\end{array}$} & $\begin{array}{l}\text { Valencia } \\
\text { semántica }\end{array}$ & \\
\hline & & & & & sub & akk \\
\hline studieren 3 & $\begin{array}{l}\text { einüben (Stilw. } \\
\text { s/v studieren). }\end{array}$ & sub akk & $\begin{array}{l}\text { jmd } \\
\text { stud } \\
\text { etwo } \\
\text { [akk }\end{array}$ & & $\begin{array}{l}+ \text { anim }] \\
{[+ \text { Mensch }]}\end{array}$ & $\begin{array}{l}\text { Lied } \\
\text { Gesangspart, } \\
\text { Rolle [-anim] } \\
\text { [-mat] } \\
\text { [+Handlung] } \\
\text { [+Konzepte] }\end{array}$ \\
\hline \multicolumn{4}{|c|}{$\begin{array}{l}\text { COSMAS II: R97/JUL.51098 Frankfurter } \\
\text { Rundschau, 03.07.1997, p. 4, Ressort: LOKAL- } \\
\text { RUNDSCHAU; "Unerkannt beim Rollenstudium } \\
\text { im Café. Daß er, anders als die ganz großen Stars } \\
\text { von Film, TV und Bühne, quasi unerkannt und } \\
\text { salopp in Jeans und Pullover im Oberurseler Cafe } \\
\text { Krämer sitzen und den Text für seine nächste Rolle } \\
\text { studieren kann, bestätigt eher sein Selbstverständnis } \\
\text { als Schauspieler, als daß sein [...]". }\end{array}$} & \multicolumn{2}{|c|}{$\begin{array}{l}\text { Posible corres- } \\
\text { pondencia en } \\
\text { español: ensayar, } \\
\text { practicar. }\end{array}$} & \\
\hline
\end{tabular}

Figura 5

\section{3. lernen}

El lexema lernen tiene tres acepciones (cfr. Fig. 6, 7, 8):

\begin{tabular}{|c|c|c|c|c|c|c|}
\hline & Significado & $\begin{array}{l}\text { Tipo de } \\
\text { actantes }\end{array}$ & \multicolumn{2}{|c|}{$\begin{array}{l}\text { Estructura } \\
\text { sintáctica }\end{array}$} & $\begin{array}{l}\text { Valencia } \\
\text { semántica }\end{array}$ & \\
\hline & & & & & sub & akk \\
\hline lernen1 & $\begin{array}{l}\text { sich ein spezielles } \\
\text { Wissen aneignen. } \\
\text { Auch } \\
\text { auswendiglernen } \\
\text { (VALBU) } \\
\text { (DUW). }\end{array}$ & $\begin{array}{l}\text { sub } \\
\text { (akk) }\end{array}$ & \multicolumn{2}{|c|}{$\begin{array}{l}\text { jmd [sub] } \\
\text { lernt } \\
\text { (etwas) } \\
\text { (akk) }\end{array}$} & $\begin{array}{l}{[+ \text { anim }]} \\
{[+ \text { Mensch }]} \\
{[+/ \text {-Tier }]}\end{array}$ & $\begin{array}{l}\text { facultativo } \\
{[+ \text { Lehrstoff }]} \\
{[\text {-anim }][- \text { mat }]} \\
\text { [+Konzept }]\end{array}$ \\
\hline \multicolumn{4}{|c|}{$\begin{array}{l}\text { COSMASII: HMP10/JAN.00117 Hamburger } \\
\text { Morgenpost, 03.01.2010, S. 14-15; "Wohin in den } \\
\text { Urlaub? Auslandsreise sollten Sie ein paar Brocken } \\
\text { der Sprache des Gastlandes lernen! So schließen } \\
\text { Sie noch schneller Kontakte. VALBU: Was ein } \\
\text { Hund zu lernen imstande ist, haben am ersten Tag } \\
\text { die Rettungshunde auf einem Parcours } \\
\text { eindrucksvoll bewiesen". (nach Mannheimer } \\
\text { Morgen, 04.10.2005). }\end{array}$} & \multicolumn{2}{|c|}{$\begin{array}{l}\text { Posible corres- } \\
\text { pondencia en } \\
\text { español: aprender }\end{array}$} & \\
\hline
\end{tabular}

Figura 6 
En oraciones con un sentido genérico o cuando se resalta la acción expresada por lernen, el akk puede ser omitido (VALBU s/v lernen).

\begin{tabular}{|c|c|c|c|c|c|c|}
\hline & Significado & $\begin{array}{l}\text { Tipo de } \\
\text { actantes }\end{array}$ & \multicolumn{2}{|c|}{$\begin{array}{l}\text { Estructura } \\
\text { sintáctica }\end{array}$} & $\begin{array}{l}\text { Valencia } \\
\text { semántica }\end{array}$ & \\
\hline & & & \multicolumn{2}{|c|}{ (2) } & sub & akk \\
\hline lernen2 & $\begin{array}{l}\text { aus etwas die } \\
\text { Notwendigkeit } \\
\text { von etwas } \\
\text { ableiten } \\
\text { (VALBU). }\end{array}$ & $\begin{array}{l}\text { sub (akk) } \\
\text { aus +Dat } \\
\text { prp }\end{array}$ & \multicolumn{2}{|c|}{$\begin{array}{l}\text { jmd [sub] } \\
\text { lernt } \\
\text { (etwas) } \\
\text { (akk) aus } \\
\text { etwas [aus } \\
\text { +Dativ] }\end{array}$} & $\begin{array}{l}{[+ \text { anim }]} \\
{[+ \text { Mensch }]} \\
{[+ \text { Tier }]}\end{array}$ & $\begin{array}{l}\text { facultativo: } \\
\text { [-anim] [-mat] } \\
\text { [+Ideen] } \\
\text { [+Zustände] } \\
\text { [+Handlungen] } \\
\text { Prp: Aus+Dat: } \\
\text { [-Mensch] } \\
\text { [-anim] [-mat] } \\
\text { [+Zustände]. }\end{array}$ \\
\hline \multicolumn{4}{|c|}{$\begin{array}{l}\text { COSMAS II:BRZ09/OKT.12838 Braunschweiger } \\
\text { Zeitung, 28.10.2009; "Und man könne aus der } \\
\text { Erfahrung lernen und diese später in den Alltag } \\
\text { mit Kindern einbauen". }\end{array}$} & \multicolumn{2}{|c|}{$\begin{array}{l}\text { Posible corres- } \\
\text { pondencia en } \\
\text { español: aprender } \\
\text { de algo }\end{array}$} & \\
\hline
\end{tabular}

Figura 7

El akk se realiza, la mayor parte de las veces como un pronombre indefinido o como una oración subordinada (VALBU s/v lernen).

\begin{tabular}{|c|c|c|c|c|c|c|}
\hline & Significado & $\begin{array}{l}\text { Tipo de } \\
\text { actantes }\end{array}$ & \multicolumn{2}{|c|}{$\begin{array}{l}\text { Estructura } \\
\text { sintáctica }\end{array}$} & $\begin{array}{l}\text { Valencia } \\
\text { semántica }\end{array}$ & \\
\hline & & & \multirow{2}{*}{\multicolumn{2}{|c|}{$\begin{array}{l}\text { jmd [sub] } \\
\text { lernt (etwas) } \\
\text { (akk) [von } \\
\text { +Dativ] }\end{array}$}} & sub & akk \\
\hline lernen3 & $\begin{array}{l}\text { von etwas } \\
\text { etwas } \\
\text { übernehmen } \\
\text { (VALBU). }\end{array}$ & $\begin{array}{l}\text { sub (akk) } \\
\text { von prp }\end{array}$ & & & $\begin{array}{l}{[+ \text { anim }]} \\
{[+ \text { Mensch }]} \\
{[+ \text { Tiere }]}\end{array}$ & $\begin{array}{l}\text { facultativo [-anim] } \\
\text { [-mat] [+Wissen] } \\
\text { [+Fertigkeit], } \\
\text { [+Verhaltensweise]; } \\
\text { prp: von+Dat: } \\
\text { [+anim] [+Mensch] } \\
\text { [+Tier] [+Ideen] }\end{array}$ \\
\hline \multicolumn{4}{|c|}{$\begin{array}{l}\text { COSMAS II: M10/JAN.07837 Mannheimer } \\
\text { Morgen, 30.01.2010, S. 10; "Und zwar auf } \\
\text { eine so effiziente und stabile Weise, dass } \\
\text { Ingenieure davon lernen können, technische } \\
\text { Systeme wie zum Beispiel Telefon- und } \\
\text { Computernetze zu verbessern”. } \\
\text { RHZ05/JUN.23412 Rhein-Zeitung, } \\
\text { 20.06.2005; "Dabei gebe es Dinge, 'die kann } \\
\text { man nicht von den Eltern lernen"'. }\end{array}$} & \multicolumn{2}{|c|}{$\begin{array}{l}\text { Posible corres- } \\
\text { pondencia en } \\
\text { español: aprender } \\
\text { de algo, aprender } \\
\text { de alguien }\end{array}$} & \\
\hline
\end{tabular}

Figura 8 


\section{Campo semántico en español}

\subsection{Practicar}

El lexema practicar presenta dos acepciones (cfr. Fig. 9, 10):

\begin{tabular}{|l|l|l|l|l|l|}
\hline & Significado & $\begin{array}{l}\text { Tipo de } \\
\text { actantes }\end{array}$ & $\begin{array}{l}\text { Estructura } \\
\text { sintáctica }\end{array}$ & $\begin{array}{l}\text { Valencia } \\
\text { semántica }\end{array}$ & \\
\hline & & & & sub & akk \\
\hline practicarl & $\begin{array}{l}\text { realizar habi- } \\
\text { tualmente } \\
\text { cierta actividad } \\
\text { que se expresa } \\
\text { con un nombre } \\
\text { (DRAE). }\end{array}$ & sub akk & $\begin{array}{l}\text { alguien } \\
{[\text { sub] practi- }} \\
\text { ca algo } \\
{[\text { akk }]}\end{array}$ & $\begin{array}{l}{[+ \text { anim }]} \\
{[+ \text { Mensch }]}\end{array}$ & $\begin{array}{l}{[\text {-anim }]} \\
{[- \text { mat }][+ \text { Handlung }]}\end{array}$ \\
\hline $\begin{array}{l}\text { CREA: El Norte de Castilla, 18/11/2002: } \\
\text { ZAMORA; "Los fines de semana suelo practi- } \\
\text { car el violín". }\end{array}$ & $\begin{array}{l}\text { Posible corres- } \\
\text { pondencia en } \\
\text { alemán: üben }\end{array}$ & \\
\hline
\end{tabular}

Figura 9

\begin{tabular}{|c|c|c|c|c|c|}
\hline & Significado & $\begin{array}{l}\text { Tipo de } \\
\text { actantes }\end{array}$ & $\begin{array}{l}\text { Estructura } \\
\text { sintáctica }\end{array}$ & $\begin{array}{l}\text { Valencia } \\
\text { semántica }\end{array}$ & \\
\hline & & & & sub & akk \\
\hline practicar2 & $\begin{array}{l}\text { ejecutar, } \\
\text { llevar a cabo } \\
\text { (María Mo- } \\
\text { liner). }\end{array}$ & sub akk & $\begin{array}{l}\text { alguien } \\
\text { [sub] prac- } \\
\text { tica algo } \\
\text { [akk] }\end{array}$ & $\begin{array}{l}{[+ \text { anim }]} \\
\text { [+Mensch }]\end{array}$ & $\begin{array}{l}{[\text {-anim }]} \\
{[- \text { mat }][+ \text { Handlung }]}\end{array}$ \\
\hline \multicolumn{3}{|c|}{$\begin{array}{l}\text { CREA: Control (La Coruña), } 1991 ; \\
\text { "Ayer por la mañana se iba a practicar la } \\
\text { autopsia". }\end{array}$} & \multicolumn{2}{|c|}{$\begin{array}{l}\text { Posible corresponden- } \\
\text { cia en alemán: durch- } \\
\text { führen, realisieren }\end{array}$} & \\
\hline
\end{tabular}

Figura 10

\footnotetext{
${ }^{8}$ Esta acepción no se encuentra dentro del campo semántico Kognition.
} 


\subsection{Estudiar}

El lexema estudiar presenta tres acepciones (cfr. Fig. 11, 12, 13):

\begin{tabular}{|c|c|c|c|c|c|c|}
\hline & Significado & $\begin{array}{l}\text { Tipo de } \\
\text { actantes }\end{array}$ & \multicolumn{2}{|c|}{$\begin{array}{l}\text { Estructura } \\
\text { sintáctica }\end{array}$} & $\begin{array}{l}\text { Valencia } \\
\text { semántica }\end{array}$ & \\
\hline & & & & & sub & akk \\
\hline estudiar1 & $\begin{array}{l}\text { Recibir ense- } \\
\text { ñanza en cierto } \\
\text { centro o de } \\
\text { cierto profe- } \\
\text { sor: cursar en } \\
\text { las universida- } \\
\text { des o en otros } \\
\text { centros docen- } \\
\text { tes (María } \\
\text { Moliner). }\end{array}$ & $\begin{array}{l}\text { sub } \\
(\mathrm{akk})\end{array}$ & \multicolumn{2}{|c|}{$\begin{array}{l}\text { alguien } \\
\text { [sub] estu- } \\
\text { dia (algo) } \\
\text { (akk) }\end{array}$} & $\begin{array}{l}\text { [+anim }] \\
{[+ \text { Mensch }]} \\
{[+ \text { Erwachsen }]}\end{array}$ & $\begin{array}{l}\text { facultativo } \\
\text { [-anim] [-mat] } \\
\text { [+Fachgebiet]; } \\
\text { sin artículo: } \\
\text { estudia dere- } \\
\text { cho [+Ideen] }\end{array}$ \\
\hline \multicolumn{4}{|c|}{$\begin{array}{l}\text { CREA: La Vanguardia, 16/11/1995; JOSEP } \\
\text { PLAYA “Quería estudiar Veterinaria, pero la } \\
\text { nota exigida [...]". }\end{array}$} & \multicolumn{2}{|c|}{$\begin{array}{l}\text { Posible correspon- } \\
\text { dencia en alemán: } \\
\text { studieren }\end{array}$} & \\
\hline
\end{tabular}

Figura 11

\begin{tabular}{|c|c|c|c|c|c|c|}
\hline & Significado & $\begin{array}{l}\text { Tipo de } \\
\text { actantes }\end{array}$ & \multicolumn{2}{|c|}{$\begin{array}{l}\text { Estructura } \\
\text { sintáctica }\end{array}$} & $\begin{array}{l}\text { Valencia } \\
\text { semántica }\end{array}$ & \\
\hline & & & & & sub & akk \\
\hline estudiar2 & $\begin{array}{l}\text { Pensar insis- } \\
\text { tentemente } \\
\text { sobre un } \\
\text { asunto para } \\
\text { resolver } \\
\text { sobre él } \\
\text { (María } \\
\text { Moliner). }\end{array}$ & sub akk & \multicolumn{2}{|c|}{$\begin{array}{l}\text { alguien } \\
\text { [sub] estu- } \\
\text { dia al- } \\
\text { go[akk] }\end{array}$} & $\begin{array}{l}{[+ \text { anim }]} \\
{[+ \text { Mensch }]}\end{array}$ & $\begin{array}{l}\text { [+anim }][+ \text { Mensch }] \\
{[+ \text { mat }][+ \text { Handlungen }]} \\
\text { [+Zustände }][+ \text { Ideen }]\end{array}$ \\
\hline \multicolumn{4}{|c|}{$\begin{array}{l}\text { CREA: La Vanguardia, 30/10/1995; "El minis- } \\
\text { tro para las Administraciones Públicas, Joan } \\
\text { Lerma, y el presidente de la Generalitat valen- } \\
\text { ciana, Eduardo Zaplana, mantendrán hoy una } \\
\text { reunión para estudiar la forma de situar a la } \\
\text { Comunidad Valenciana en el mismo techo } \\
\text { competencial que las comunidades históricas.- } \\
\text { Europa Press". }\end{array}$} & \multicolumn{2}{|c|}{$\begin{array}{l}\text { Posible corres- } \\
\text { pondencia en } \\
\text { alemán: prüfen, } \\
\text { untersuchen }\end{array}$} & \\
\hline
\end{tabular}

Figura 12 


\begin{tabular}{|c|c|c|c|c|c|c|}
\hline & Significado & $\begin{array}{l}\text { Tipo de } \\
\text { actantes }\end{array}$ & \multicolumn{2}{|c|}{$\begin{array}{l}\text { Estructura } \\
\text { sintáctica }\end{array}$} & $\begin{array}{l}\text { Valencia } \\
\text { semántica }\end{array}$ & \\
\hline & & & & & sub & akk \\
\hline estudiar3 & $\begin{array}{l}\text { particularmente, } \\
\text { leer atentamente } \\
\text { lo que dice un } \\
\text { libro sobre cierta } \\
\text { materia, para } \\
\text { aprenderlo. } \\
\text { (DRAE). }\end{array}$ & sub akk & $\begin{array}{l}\text { apre } \\
\text { (ton } \\
\text { mer } \\
\text { algu } \\
\text { [sub } \\
\text { dia } \\
\text { [akl }\end{array}$ & $\begin{array}{l}\text { der } \\
\text { ar de } \\
\text { oria) } \\
\text { en } \\
\text { estu- } \\
\text { go }\end{array}$ & $\begin{array}{l}{[+ \text { anim }]} \\
{[+ \text { Mensch }]} \\
{[- \text { Tier }]}\end{array}$ & $\begin{array}{l}\text { [+anim][-mat] } \\
{[+ \text { Konzepte }]}\end{array}$ \\
\hline \multicolumn{4}{|c|}{$\begin{array}{l}\text { CREA: El Mundo, 07/02/1995; } \\
\text { "Desde hace quince días vive encerrado en una } \\
\text { casa alquilada con piscina que "todavía he utili- } \\
\text { zado poco" de un suburbio residencial de esta } \\
\text { ciudad, enfrascado en estudiar su nuevo papel". }\end{array}$} & \multicolumn{2}{|c|}{$\begin{array}{l}\text { Posible corres- } \\
\text { pondencia en } \\
\text { alemán: auswen- } \\
\text { diglernen }\end{array}$} & \\
\hline
\end{tabular}

\section{Figura 13}

\section{3. aprender}

El lexema aprender presenta dos acepciones (Fig. 14, 15):

\begin{tabular}{|c|c|c|c|c|c|c|}
\hline & Significado & $\begin{array}{l}\text { Tipo de } \\
\text { actantes }\end{array}$ & \multicolumn{2}{|c|}{$\begin{array}{l}\text { Estructura } \\
\text { sintáctica }\end{array}$} & $\begin{array}{l}\text { Valencia } \\
\text { semántica }\end{array}$ & \\
\hline & & & & & sub & akk \\
\hline aprenderl & $\begin{array}{l}\text { adquirir el } \\
\text { conocimiento } \\
\text { de algo por } \\
\text { medio del } \\
\text { estudio o de la } \\
\text { experiencia } \\
\text { (DRAE). }\end{array}$ & $\begin{array}{l}\text { sub } \\
(\mathrm{akk})\end{array}$ & \multicolumn{2}{|c|}{$\begin{array}{l}\text { Alguien } \\
\text { [sub] apren- } \\
\text { de (algo) } \\
\text { (akk) }\end{array}$} & $\begin{array}{l}+ \text { anim }] \\
{[+ \text { Mensch }]} \\
{[+ \text { Tier }]}\end{array}$ & $\begin{array}{l}\text { facultativo } \\
\text { [+Lehrstoff] } \\
\text { [-anim] } \\
\text { [-mat] } \\
\text { [+Konzepte] } \\
\text { [+Ideen] } \\
\text { [+Handlungen] }\end{array}$ \\
\hline \multicolumn{4}{|c|}{$\begin{array}{l}\text { CREA: El Mundo - Su Ordenador (Suplemen- } \\
\text { to), 06/04/1997; "Los niños, a esta edad, co- } \\
\text { mienzan a aprender informática". }\end{array}$} & \multicolumn{2}{|c|}{$\begin{array}{l}\text { Posible corres- } \\
\text { pondencia en } \\
\text { alemán: lernen }\end{array}$} & \\
\hline
\end{tabular}

\section{Figura 14}

Ejemplo: "Vamos a aprender de este ejemplo": se encuentra en el corpus del español de Mark Davies, (http://zedillo.presidencia.gob.mx/ el corpus del español de Mark Davies). "Debías aprender de nosotros" (Rayuela, Julio Cortázar (213, 210)). No hay ninguna acepción en los diccionarios españoles consultados que corresponda a estos ejemplos donde se refleja el uso preposicional "aprender de". 


\begin{tabular}{|c|c|c|c|c|c|c|}
\hline & Significado & $\begin{array}{l}\text { Tipo de } \\
\text { actantes }\end{array}$ & \multicolumn{2}{|c|}{$\begin{array}{l}\text { Estructura } \\
\text { sintáctica }\end{array}$} & $\begin{array}{l}\text { Valencia } \\
\text { semántica }\end{array}$ & \\
\hline & & & & & sub & akk \\
\hline aprender2 & $\begin{array}{l}\text { Fijar algo en la } \\
\text { memoria. To- } \\
\text { mar algo en la } \\
\text { memoria } \\
\text { (DRAE). }\end{array}$ & sub akk & \multicolumn{2}{|c|}{$\begin{array}{l}\text { Alguien } \\
\text { [sub] apren- } \\
\text { de algo } \\
\text { [akk] }\end{array}$} & $\begin{array}{l}{[+ \text { anim }]} \\
{[+ \text { Mensch }]}\end{array}$ & $\begin{array}{l}{[\text {-anim }]} \\
{[\text {-mat }][+ \text { Konzept }]}\end{array}$ \\
\hline \multicolumn{4}{|c|}{$\begin{array}{l}\text { CREA: La Vanguardia, 15/09/1994; “[...] pero sí } \\
\text { 'lecciones para aprender ', en un intento explícito } \\
\text { de "rehabilitación de la memoria" y de preparar- } \\
\text { los". }\end{array}$} & \multicolumn{2}{|c|}{$\begin{array}{l}\text { Posible corres- } \\
\text { pondencia en } \\
\text { alemán: aus- } \\
\text { wendiglernen }\end{array}$} & \\
\hline
\end{tabular}

Figura 15

\section{Análisis contrastivo alemán-español-alemán}

A estos análisis hay que añadir el análisis contrastivo alemán-español. En un primer lugar, presentaré el contraste de las equivalencias alemán español / españolalemán: en segundo lugar mostraré el análisis contrastivo de las valencias semánticas de los lexemas, así como la de sus semas más relevantes. El análisis contrastivo está hecho en base a las acepciones de los lexemas elegidos (cfr. Fig. 16):

\begin{tabular}{|l|l|}
\hline üben1 & practicar1 \\
\hline üben2 & practicar1 \\
\hline studieren1 & estudiar1 \\
\hline studieren2 & estudiar2 \\
\hline studieren3 & practicar1 \\
\hline lernen1 & aprender1, aprender2, estudiar3 \\
\hline lernen2 & aprender1 \\
\hline lernen3 & aprender1 \\
\hline
\end{tabular}

Figura 16

Como se ve en este esquema, sólo es posible realizar un análisis contrastivo válido, si se tienen en cuenta todas las acepciones de los lexemas. En este sentido, se puede observar un comportamiento bastante paralelo en las dos lenguas, con alguna diferencia: las dos acepciones en alemán de üben corresponden en español a practicar; studieren3: (Akten, Rolle, Gesangspart studieren) corresponde, no obstante, a praticarl (y no a estudiar3), en el sentido de practicar, ensayar.

En cuanto a lernen, lernen1 cubre el contenido semántico de aprender1, aprender 2 y estudiar 3 , es decir, aprender en sentido general y aprender de memoria. Los usos preposicionales lernen 2 y lernen 3 corresponden a aprenderl ya que, 
en español, no se tiene en cuenta el uso preposicional, aunque sí que han aparecido ejemplos de este tipo, como se ha visto anteriormente.

En cuanto al análisis del campo semántico español-alemán, tenemos el siguiente esquema (cfr. Fig. 17):

\begin{tabular}{|l|l|}
\hline practicar1 & üben1, üben2, studieren3 \\
\hline practicar2 & (durchführen, realisieren) \\
\hline estudiar1 & studieren1 \\
\hline estudiar2 & studieren2 \\
\hline estudiar3 & lernen1 \\
\hline aprender1 & lernen1, lernen2, lernen3 \\
\hline aprender2 & lernen1 \\
\hline
\end{tabular}

Figura 17

Practicar 1 corresponde en alemán a üben 1, üben 2 y studieren 3 (practicar, ensayar). Según se ha comentado anteriormente, practicar2 no se encuentra dentro del campo semántico que nos ocupa, ya que corresponde a otras acepciones (durchführen, realisieren). Estudiar3 (estudiar de memoria) tiene como equivalencia lernen 1 en el sentido (auswendiglernen). Aprenderl corresponde a las tres acepciones en alemán: lernen1, lernen2, lernen3, ya que, en alemán, sí que existe en los diccionarios la diferenciación del uso preposicional: lernen1, en sentido general y lernen2, lernen 3: aprender de algo, aprender de alguien. Aprender 2 se corresponde, sin embargo con lernen 1 (auswendiglernen).

En la tabla siguiente (cfr. Figura 18) se han contrastado cada uno de los rasgos semánticos de las acepciones de los lexemas analizados. Con ello se puede observar que, al comparar las diferentes acepciones en alemán y español, éstas pueden diferir en cuanto a los rasgos semánticos que presentan. Los semas considerados son los siguientes: S1: [lesen]; S2: [theoretische Kenntnisse]; S3: [iterativ]; S4: [Universität]; S5: [Gedächtnis]; S6: [Standardsprache]:

\begin{tabular}{|l|l|l|l|l|l|l|}
\hline & $\mathbf{S 1}$ & $\mathbf{S 2}$ & $\mathbf{S 3}$ & $\mathbf{S 4}$ & $\mathbf{S 5}$ & $\mathbf{S 6}$ \\
\hline üben 1 & {$[+/-]$} & {$[-]$} & {$[+]$} & {$[+/-]$} & {$[+/-]$} & {$[+]$} \\
üben2 & {$[+/-]$} & {$[-]$} & {$[+]$} & {$[+/-]$} & {$[+/-]$} & {$[-]$} \\
Practicar1 & {$[+/-]$} & {$[-]$} & {$[+]$} & {$[+/-]$} & {$[+/-]$} & {$[+]$} \\
\hline atudieren 1-2-3 & {$[+][+]$} & {$[+][+/-]$} & {$[-][-]$} & {$[+][+/-]$} & {$[+/-][-]$} & {$[+]$} \\
estudiar 1-2- & {$[+/-]$} & {$[-]$} & {$[+]$} & {$[+/-]$} & {$[+/-]$} & {$[+/-]^{10}$} \\
practicar1 & {$[+]$} & {$[+]$} & {$[-]$} & {$[+]$} & {$[+/-]$} & {$[+]$} \\
& {$[+]$} & {$[+/-]$} & {$[-]$} & {$[+/-]$} & {$[-]$} & {$[+]$} \\
& {$[+/-]$} & {$[-]$} & {$[+]$} & {$[+/-]$} & {$[+/-]$} & {$[+][+]$} \\
\hline lernen 1-2-3 & {$[+/-]$} & {$[+/-]$} & {$[-]$} & {$[+/-]$} & {$[-]$} & {$[+]$} \\
\hline
\end{tabular}

\footnotetext{
${ }^{9}$ La segunda acepción de üben tiene un registro lingüístico propio de la lengua elevada o culta (véase üben2).

${ }^{10}$ La acepción studieren 2 tiene una variante coloquial: sie studierten eigehend die Speisekarte, im Sinn von lasen sie genau durch (STILW. s/v studieren).
} 


\begin{tabular}{|l|l|l|l|l|l|l|}
\hline aprender 1 & {$[+/-]$} & {$[+/-]$} & {$[-]$} & {$[+/-]$} & {$[-]$} & {$[+]$} \\
& {$[+/-]$} & {$[+/-]$} & {$[-]$} & {$[+/-]$} & {$[-]$} & {$[+]$} \\
& {$[+/-]$} & {$[+/-]$} & {$[-]$} & {$[+/-]$} & {$[-]$} & {$[+]$} \\
\hline lernen 1 & {$[+]$} & {$[+]$} & {$[+]$} & {$[+/-]$} & {$[+]$} & {$[+]$} \\
aprender2 & {$[+]$} & {$[+]$} & {$[+]$} & {$[+/-]$} & {$[+]$} & {$[+]$} \\
estudiar 3 & {$[+]$} & {$[+]$} & {$[+]$} & {$[+/-]$} & {$[+]$} & {$[+]$} \\
\hline
\end{tabular}

Figura 18

En virtud del análisis contrastivo de rasgos semánticos, se puede observar que los pares contrastados actúan de manera bastante paralela. Sólo hay diferenciación (sombreado en gris) en los casos en que hay alguna variante estilística sobre el registro lingüístico.

En el análisis español-alemán se han variado ligeramente algunos semas, con el fin de llegar a un mayor acercamiento del significado (cfr. Figura 19): S1: [lesen]; S2: [praktisch]; S3: [iterativ]; S4: [Universität]; S5: [Gedächtnis]; S6: [Standardsprache]; S7:[mechanisch]. El resultado del contraste de las acepciones de los lexemas es el siguiente:

\begin{tabular}{|c|c|c|c|c|c|c|c|}
\hline & S1 & S2 & S3 & S4 & S5 & S6 & S7 \\
\hline practicar 1 & {$[+/-]$} & {$[+]$} & {$[+]$} & {$[+/-]$} & {$[+/-]$} & {$[+]$} & {$[-]$} \\
\hline üben 1 & {$[+/-]$} & {$[+]$} & {$[+]$} & {$[+/-]$} & {$[+/-]$} & {$[+]$} & {$[-]$} \\
\hline üben2 & {$[+/-]$} & {$[+]$} & {$[+]$} & {$[+/-]$} & {$[+/-]$} & {$[-]^{11}$} & {$[-]$} \\
\hline atudieren 3 & {$[+/-]$} & {$[+]$} & {$[+]$} & {$[+/-]$} & {$[+/-]$} & {$[+]$} & {$[-]$} \\
\hline \multirow{2}{*}{$\begin{array}{l}\text { estudiar } 1-2 \\
\text { studieren } 1-2\end{array}$} & {$[+][+]$} & {$[-][-]$} & {$[-][-]$} & {$[+][+/-]$} & {$[-][-]$} & {$[+][+]$} & {$[-][-]$} \\
\hline & {$[+][+]$} & {$[-][-]$} & {$[-][-]$} & {$[+][+/-]$} & {$[-][-]$} & {$[+][+/-]^{12}$} & {$[-][-]$} \\
\hline \begin{tabular}{|l|} 
estudiar \\
\end{tabular} & {$[+]$} & {$[-]$} & {$[+]$} & {$[+/-]$} & {$[+]$} & {$[+]$} & {$[+]$} \\
\hline lernen 1 & {$[+]$} & {$[-]$} & {$[+]$} & {$[+/-]$} & {$[+]$} & {$[+]$} & {$[+]$} \\
\hline \begin{tabular}{|l} 
aprender 1 \\
\end{tabular} & {$[+/-]$} & {$[+/-]$} & {$[-]$} & {$[+/-]$} & {$[-]$} & {$[+]$} & {$[+/-]$} \\
\hline lernen 1 & {$[+/-]$} & {$[+/-]$} & {$[-]$} & {$[+/-]$} & {$[-]$} & {$[+]$} & {$[+/-]$} \\
\hline lernen 2 & {$[+/-]$} & {$[+/-]$} & {$[-]$} & {$[+/-]$} & {$[-]$} & {$[+]$} & {$[-]$} \\
\hline lernen 3 & {$[+/-]$} & {$[+/-]$} & {$[-]$} & {$[+/-]$} & {$[-]$} & {$[+]$} & {$[-]$} \\
\hline aprender 2 & {$[+]$} & {$[-]$} & {$[+]$} & {$[+/-]$} & {$[+]$} & {$[+]$} & {$[+]$} \\
\hline lernen 1 & {$[+]$} & {$[-]$} & {$[+]$} & {$[+/-]$} & {$[+]$} & {$[+]$} & {$[+]$} \\
\hline
\end{tabular}

Figura 19

Se ha omitido la acepción practicar2 (durchführen realisieren), ya que queda fuera del campo semántico Kognition. Las únicas divergencias en el contraste españolalemán corresponden, como se puede ver, a: 1 . diferencias en el registro lingüístico, como ocurría en el análisis alemán-español; 2. Existen también diferencias entre las acepciones aprender1 y lernen1, lernen2, lernen3: desde mi punto de vista, el lexe-

11 Sich üben in: lengua culta.

${ }^{12}$ Véase nota 9. 
ma aprender (en sentido general) puede poseer el sema [mechanisch], pero no lo tienen las acepciones lernen2 y lernen3 (aprender de algo o de alguien), lo cual no supone una acción en modo alguno mecánica. Por lo demás, el análisis de rasgos semánticos ofrece también un resultado paralelo en las dos lenguas.

Veamos cómo funciona el análisis contrastivo de los actantes de cada una de las acepciones (cfr. Figura 20); primero presentaré el análisis alemán-español y después el análisis español-alemán. Añado, además, la valencia semántica de los actantes $^{13}$ :

\begin{tabular}{|c|c|c|c|}
\hline & $\begin{array}{l}\text { sub } \\
\text { Wer? } \\
\text { Was? }\end{array}$ & $\begin{array}{l}\text { akk } \\
\text { Wen? } \\
\text { Was? }\end{array}$ & Prp \\
\hline $\begin{array}{l}\text { üben } 1 \\
\text { practicarl }\end{array}$ & $\begin{array}{l}+ \text { Mensch }][+ \text { anim }] \\
{[+ \text { Tier }][+/ \text {-erwachsen }]} \\
\\
{[+ \text { anim }]} \\
{[+ \text { Mensch }][+/ \text {-erwachsen }]} \\
{[- \text { Tier }]}\end{array}$ & $\begin{array}{l}\text { Fac } \\
{[\text {-anim][-mat }]} \\
{[+ \text { Zustand }]} \\
{[+ \text { Handlung }]} \\
{[\text {-anim][-mat }]} \\
\text { [+Zustand] } \\
\text { [+Handlung] }\end{array}$ & \\
\hline $\begin{array}{l}\text { üben2 } \\
\text { practicar } 1\end{array}$ & $\begin{array}{l}{[+ \text { anim }]} \\
{[+ \text { Mensch }][+ \text {-erwachsen }]} \\
{[+ \text { Tier }]} \\
{[+ \text { anim }]} \\
{[+/ \text {-erwachsen }]} \\
{[+ \text { Mensch }][- \text { Tier }]}\end{array}$ & $\begin{array}{l}\text { En alemán funciona sin } \\
\text { acusativo } \\
{[\text {-anim] }} \\
{[\text {-mat }][+ \text { Handlung }]}\end{array}$ & $\begin{array}{l}\text { In+ Dativ } \\
\text { [-anim] } \\
\text { [-mat] } \\
\text { [+Handlung] }\end{array}$ \\
\hline estudiar1 & $\begin{array}{l}{[+ \text { anim }][+ \text { Mensch }]} \\
{[- \text { Tier }][+ \text { erwachsen }]} \\
\\
{[+ \text { anim }]} \\
{[+ \text { Mensch }][- \text { Tier }]} \\
{[+ \text { erwachsen }]}\end{array}$ & $\begin{array}{l}\text { Fac } \\
\text { [-anim] } \\
\text { [-mat] } \\
\text { [+Fachgebiet] sin } \\
\text { artículo: sie studiert } \\
\text { Chemie [+Ideen] } \\
\text { Fac } \\
\text { [-anim] } \\
\text { [-mat] } \\
\text { [+Fachgebiet][+Ideen] } \\
\text { Estudia derecho }\end{array}$ & \\
\hline studieren 2 & $\begin{array}{l}{[+ \text { anim }][+ \text { Mensch }]} \\
{[- \text { Tier }][+ \text { erwachsen }]}\end{array}$ & $\begin{array}{l}{[+ \text { anim }][+ \text { Mensch }]} \\
{[+ \text { mat }] \text { (Akten) }} \\
{[+ \text { Handlung }]} \\
\text { [+Zustand }] \\
\text { [+Ideen }]\end{array}$ & \\
\hline
\end{tabular}

${ }^{13}$ Los semas que se han tenido en cuenta en el análisis son los siguientes: [+Mensch] [+anim]: Lebewesen; [+Tier] [+erwachsen] [-mat]: materiell; [+Zustand] [+Handlung] [+Fachgebiet] [+Ideen] [+Konzept] [+Lehrstoff] [+Wissen] [+Fertigkeit] [+Verhaltensweise]. 


\begin{tabular}{|c|c|c|c|}
\hline estudiar2 & $\begin{array}{l}{[+ \text { anim }][+ \text { Mensch }]} \\
{[- \text { Tier }][+ \text { erwachsen }]}\end{array}$ & $\begin{array}{l}{[\underline{+} \text { anim }]} \\
{[+ \text { Mensch }]} \\
{[+ \text { mat }]} \\
{[+ \text { Handlung }]} \\
\text { [+Zustand }] \\
{[+ \text { Ideen }]}\end{array}$ & \\
\hline studieren 3 & $\begin{array}{l}{[+ \text { anim }][+ \text { Mensch }]} \\
{[- \text { Tier }][+/ \text {-erwachsen }]}\end{array}$ & $\begin{array}{l}{[\text {-anim][-mat }]} \\
{[+ \text { Handlung] }} \\
\text { [+Konzept }]\end{array}$ & \\
\hline $\begin{array}{l}\text { practicarl } \\
\text { ensayar }\end{array}$ & $\begin{array}{l}{[+ \text { anim }][+ \text { Mensch }]} \\
{[- \text { Tier }][+/ \text {-erwachsen }]}\end{array}$ & $\begin{array}{l}\text { [-anim] } \\
{[- \text { mat }]} \\
\text { [+Konzept }] \\
\text { [+Handlung }]\end{array}$ & \\
\hline $\begin{array}{l}\text { lernen1 } \\
\text { aprender1 }\end{array}$ & $\begin{array}{l}{[+ \text { anim }][+ \text { Mensch }][+ \text { Tier }]} \\
{[+/ \text {-erwachsen }]} \\
{[+ \text { anim }][+ \text { Mensch }][+ \text { Tier }]} \\
{[+/ \text {-erwachsen }]}\end{array}$ & $\begin{array}{l}\text { Fac } \\
\text { [+Lehrstoff][-anim] } \\
\text { [-mat][Konzept] } \\
\text { [Handlung] } \\
\text { Fac.[+Lehrstoff] } \\
\text { [-anim][-mat] } \\
\text { [+Konzept] } \\
\text { [+Ideen] } \\
\text { [+Handlung] }\end{array}$ & \\
\hline $\begin{array}{l}\text { lernen1 } \\
\text { aprender2 }\end{array}$ & $\begin{array}{l}{[+ \text { anim }][+ \text { Mensch }]} \\
{[- \text { Tier }]} \\
{[+/ \text {-erwachsen }]} \\
\\
{[+ \text { anim }][+ \text { Mensch }]} \\
{[- \text { Tier }]} \\
{[+/ \text {-erwachsen }]}\end{array}$ & $\begin{array}{l}{[- \text { anim }]} \\
{[\text {-mat }]} \\
{[+ \text { Konzept }]} \\
{[+ \text { Lehrstoff }]} \\
{[\text {-anim }]} \\
{[\text {-mat }]} \\
\text { [+Konzept }] \\
{[+ \text { Lehrstoff }]}\end{array}$ & \\
\hline $\begin{array}{l}\text { lernen1 } \\
\text { estudiar3 }\end{array}$ & $\begin{array}{l}{[+ \text { anim }][+ \text { Mensch }]} \\
{[- \text { Tier }]} \\
{[+/ \text {-erwachsen }]} \\
{[+ \text { anim }]} \\
{[+ \text { Mensch }][- \text { Tier }]} \\
{[+/ \text {-erwachsen }]}\end{array}$ & $\begin{array}{l}{[\text {-anim] }} \\
\text { [-mat] } \\
{[+ \text { Konzept }]} \\
{[+ \text { Lehrstoff }]} \\
{[\text {-anim }]} \\
{[- \text {-mat }]} \\
{[+ \text { Konzept }]} \\
{[+ \text { Lehrstoff }]}\end{array}$ & \\
\hline lernen2 & $\begin{array}{l}{[+ \text { anim }][+ \text { Mensch }]} \\
{[+ \text { Tier }]} \\
{[+/ \text {-erwachsen }]}\end{array}$ & $\begin{array}{l}\text { Fac } \\
\text { [-anim][-mat] } \\
\text { [+Ideen] } \\
\text { [+Zustand] } \\
\text { [+Handlung] }\end{array}$ & $\begin{array}{l}\text { aus + Dativ } \\
{[\text {-anim] }} \\
{[- \text { mat }]} \\
\text { [-Mensch] } \\
\text { [+Zustand }]\end{array}$ \\
\hline
\end{tabular}




\begin{tabular}{|c|c|c|c|}
\hline aprender1 & $\begin{array}{l}{[+ \text { anim }][+ \text { Mensch }]} \\
{[+ \text { Tier }]} \\
{[+/ \text {-erwachsen }]}\end{array}$ & $\begin{array}{l}\text { Fac } \\
\text { [+Lehrstoff][-anim }] \\
{[- \text { mat }]} \\
{[+ \text { Konzept }][+ \text { Ideen }]}\end{array}$ & \\
\hline $\begin{array}{l}\text { lernen3 } \\
\text { aprender } 1\end{array}$ & $\begin{array}{l}{[+ \text { anim }][+ \text { Mensch }]} \\
{[+ \text { Tier }]} \\
\text { [+/-erwachsen }] \\
\\
{[+ \text { anim }]} \\
{[+ \text { Mensch }][+ \text { Tier }]} \\
\text { [+/-erwachsen }]\end{array}$ & $\begin{array}{l}\text { Fac } \\
\text { [-anim][-mat] } \\
\text { [+Wissen] } \\
\text { [+Fertigkeit] } \\
\text { [+Verhaltensweise] } \\
\text { Fac[+Lehrstoff] } \\
\text { [-anim] } \\
\text { [-mat]+Konzept] } \\
\text { [+Ideen] } \\
\text { [+Handlung] }\end{array}$ & $\begin{array}{l}\text { Von+Dativ } \\
{[+ \text { anim] }} \\
\text { [+Mensch] } \\
\text { [+Ideen] } \\
\text { [+Tier] }\end{array}$ \\
\hline
\end{tabular}

Figura 20

Uno de los aspectos, que se deben resaltar en el análisis alemán-español, lo constituye el carácter facultativo del acusativo (akk), que suele coincidir en la mayoría de los casos ${ }^{14}$. Algunos verbos no llevan akk como üben2 (sich üben in). La presencia del rasgo [+Tier] en el sujeto, en español, no existe para el lexema practicarl, ya que no se han encontrado ejemplos de esta construcción. En alemán, sí que existen ejemplos para übenl con el rasgo [+Tier]. El rasgo [-Tier] vuelve a aparecer en el lexema lernen 1 con la acepción auswendiglernen y, por tanto, también para aprender 2 y estudiar3. Las acepciones studieren1-estudiar1 y studieren2-estudiar 2 también se caracterizan por llevar el rasgo [-Tier] en el sujeto. El comportamiento de los lexemas desde el punto de vista contrastivo es bastante paralelo y apenas se pueden reseñar otras diferencias relevantes.

El análisis español-alemán queda como sigue (cfr. Figura 21):

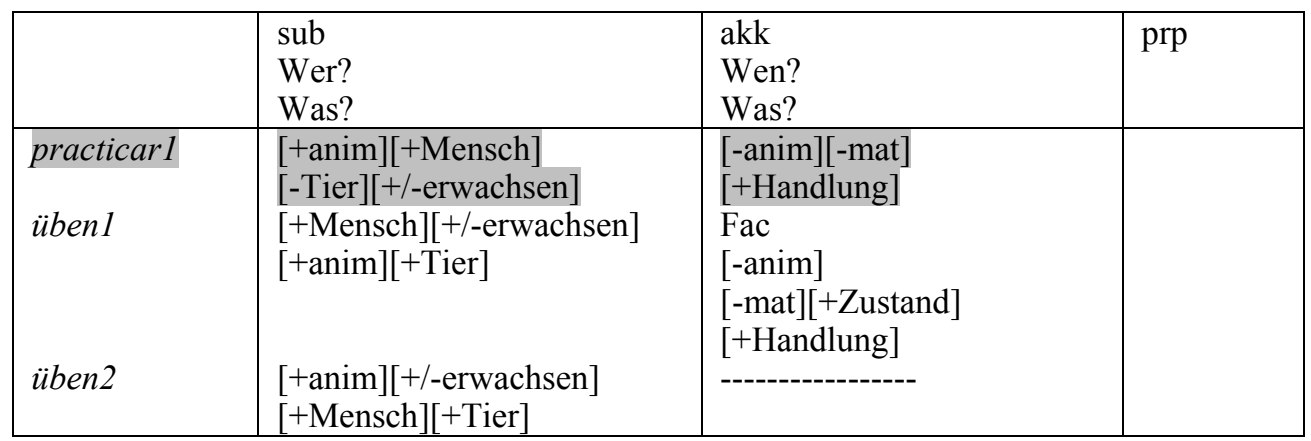

${ }^{14}$ Un caso donde no hay coincidencia lo constituye el par üben1/practicarl, donde, en alemán, el akk está documentado como facultativo y en español, el complemento directo, no. 


\begin{tabular}{|c|c|c|c|}
\hline studieren3 & $\begin{array}{l}{[\text {-anim }][+ \text { Mensch }]} \\
{[\text {-Tier }][+/ \text {-erwachsen }]}\end{array}$ & $\begin{array}{l}\text { [-anim }][-\mathrm{mat}] \\
{[+ \text { Konzept }][+ \text { Handlung }]}\end{array}$ & \\
\hline estudiarl & $\begin{array}{l}{[+ \text { anim }][+ \text { Mensch }]} \\
{[+ \text { erwachsen }][- \text { Tier }]}\end{array}$ & $\begin{array}{l}\text { Fac } \\
{[\text {-anim }][- \text { mat }]} \\
[+ \text { Fachgebiet }] \text { [+Ideen }]\end{array}$ & \\
\hline studieren 1 & $\begin{array}{l}{[+ \text { anim }][+ \text { Mensch }]} \\
{[+ \text { erwachsen }][- \text { Tier }]}\end{array}$ & $\begin{array}{l}\text { Fac. }[\text {-anim }][\text {-mat }] \\
{[+ \text { Fachgebiet }][+ \text { Ideen }]}\end{array}$ & \\
\hline estudiar 2 & $\begin{array}{l}{[+ \text { anim }][+ \text { Mensch }]} \\
{[\text {-Tier }][+ \text { erwachsen }]}\end{array}$ & $\begin{array}{l}{[\text {-anim][-mat] }} \\
{[+ \text { Konzept }]}\end{array}$ & \\
\hline studieren 2 & $\begin{array}{l}{[+ \text { anim }][+ \text { Mensch }]} \\
{[- \text { Tier }][+ \text { erwachsen }]}\end{array}$ & $\begin{array}{l}{[- \text { anim }][- \text { mat }]} \\
{[+ \text { Konzept }]}\end{array}$ & \\
\hline estudiar3 & $\begin{array}{l}{[+ \text { anim }][+ \text { Mensch }]} \\
{[- \text { Tier }][+/ \text {-erwachsen }]}\end{array}$ & $\begin{array}{l}{[\text {-anim }][-\mathrm{mat}]} \\
{[+ \text { Konzept }]}\end{array}$ & \\
\hline lernen 1 & $\begin{array}{l}{[+ \text { anim }][+ \text { Mensch }]} \\
{[- \text { Tier }][+/ \text {-erwachsen }]}\end{array}$ & $\begin{array}{l}\text { [+Lehrstoff }] \\
{[\text {-anim }][\text {-mat }][\text { Konzept }]}\end{array}$ & \\
\hline aprenderl & $\begin{array}{l}{[+ \text { anim }][+ \text { Mensch }]} \\
{[+ \text { Tier }][+/ \text {-erwachsen }]} \\
{[+ \text { anim }][+ \text { Mensch }]} \\
{[+ \text { Tier }][+/ \text {-erwachsen }]}\end{array}$ & $\begin{array}{l}\text { Fac[-anim][-mat] } \\
\text { [+Konzept] } \\
\text { [+Lehrstoff] } \\
\text { Fac[-anim][-mat }] \\
\text { [+Konzept][+Lehrstoff] }\end{array}$ & \\
\hline lernen2 & $\begin{array}{l}{[+ \text { anim }][+ \text { Mensch }]} \\
{[+ \text { Tier }][+/ \text {-erwachsen }]}\end{array}$ & $\begin{array}{l}\text { Fac }[\text {-anim }][- \text { mat }] \\
{[+ \text { Konzept }][+ \text { Lehrstoff }}\end{array}$ & $\begin{array}{l}\text { Aprender } \\
\text { algo de } \\
\text { /sobre }\end{array}$ \\
\hline lernen 3 & $\begin{array}{l}{[+ \text { anim }][+ \text { Mensch }]} \\
{[+ \text { Tier }][+/ \text {-erwachsen }]}\end{array}$ & $\begin{array}{l}\text { Fac }[\text {-anim }][- \text { mat }] \\
{[+ \text { Konzept }][+ \text { Lehrstoff }]}\end{array}$ & $\begin{array}{l}\text { Alguien/ } \\
\text { Algo }\end{array}$ \\
\hline $\begin{array}{l}\text { aprender2 } \\
\text { lernen1 }\end{array}$ & $\begin{array}{l}{[+ \text { anim }][+ \text { Mensch }]} \\
{[- \text { Tier }][+ \text {--erwachsen }]} \\
{[+ \text { anim }][+ \text { Mensch }]} \\
{[- \text { Tier }][+/ \text {-erwachsen }]}\end{array}$ & $\begin{array}{l}\text { [-anim }][\text {-mat }] \\
\text { [+Konzept][+Lehrstoff }] \\
\text { [-anim }][- \text { mat }] \\
\text { [+Konzept }][+ \text { Lehrstoff }]\end{array}$ & \\
\hline
\end{tabular}

Figura 21

En el análisis español-alemán se observan características similares que en el análisis alemán-español. En español no aparece documentada la acepción "aprender de alguien o de algo", aunque sí que se ha encontrado algún ejemplo en el corpus sobre estos usos.

\section{Conclusión}

De este análisis se desprenden una serie de conclusiones que surgen al contrastar la lengua alemana y la española: 
Desde el punto de vista semántico:

1. En algunos casos, algunas acepciones de los lexemas no entran dentro del campo semántico Kognition, véase practicar $2 \rightarrow$ realisieren, durchführen, o bien practicarl o üben 1 en los casos en los que estos verbos no están relacionados con la realización de una actividad mental, por ejemplo practicar deporte.

2. En otros casos, las posibilidades combinatorias de ciertos verbos son muy limitadas y no existe una correspondencia directa en la otra lengua: studieren $3 \rightarrow$ no es estudiar sino ensayar, practicar y se limita a sustantivos como Rolle Lied, Gesangspart. En alemán, este verbo muestra una capacidad combinatoria menor que su equivalencia en español, ya que en español, practicar tiene un sentido más general, mientras que en alemán, el sentido practicar se limita a las colocaciones Rolle, Lied Gesangspart studieren. Habría que decir que el alemán es, en este sentido, más preciso que el español, por ello necesita colocaciones más específicas.

3. El rasgo semántico [ + Tier] puede cambiar en el sujeto para el alemán y para el español, dependiendo del verbo de que se trate. Normalmente suele ser positivo [+ Tier] en alemán y negativo en español [- Tier], lo que demuestra que el contenido semántico es mayor para el alemán que para el español.

Desde el punto de vista sintáctico:

1. La mayoría de las divergencias reside en la existencia del acusativo/ complemento directo como facultativo u obligatorio en una lengua y otra.

2. En los diccionarios españoles, destaca la dificultad de documentar la información sintagmática. Así, por ejemplo, no se ha encontrado, para aprenderl, ninguna acepción que haga alusión al uso preposicional: aprender de alguien o de algo. No obstante, sí que han aparecido ejemplos sobre estos usos en el corpus de COSMAS II. Esto refleja una gran diferencia entre la lengua registrada en los diccionarios y los usos reales de la misma. De aquí se desprende la necesidad de nuevos trabajos lexicográficos que incluyan este tipo de información. En la actualidad, y, en el marco de la lexicografía bilingüe alemán-español, no existe ninguna obra de este tipo, tan sólo proyectos o estudios parciales del léxico.

Después de realizar este análisis contrastivo, se puede afirmar que, aunque no existen demasiadas diferencias entre el campo semántico en alemán y en español, éstas son menores en el plano sintáctico que en el semántico. Como se ha observado, este tipo de análisis sólo tiene verdadero sentido si se realiza a partir de las acepciones de los lexemas y no a partir de los lexemas mismos. La utilización de ejemplos reales procedentes de periódicos en alemán y en español documentan las acepciones encontradas en los diccionarios. Esto refleja, del mismo modo, las divergencias entre la lengua normalizada y su uso real. La relación entre este tipo de análisis y la práctica lexicográfica sería, pues, materia y objeto de discusión para otro trabajo propio, según se desprende de la literatura citada. Resulta inne- 
gable, no obstante, la ayuda inestimable de los diccionarios para la elaboración de tal tipo de estudios, los cuales, además, sirven para incrementar la competencia lingüística y la comprensión de la lengua extranjera al contrastarla con la lengua materna. La creación de nuevos diccionarios bilingües exigen hoy en día nuevos retos, como son, entre otros, la fundamentación de éstos en estudios lexicológicos sólidos y actuales y la inclusión de la información sintagmática, aparte de la paradigmática, con el fin de servir de apoyo al usuario no sólo en la recepción, sino también en la producción de textos.

\section{Referencias bibliográficas}

Berlin-Branderburgische AKADEMIE DER WiSSENSCHAFTEN, Digitales Wörterbuch der deutschen Sprache (DWDS) Projekt, http://www.dwds.de. 2008-2011 [15/10/ 2011].

CANOONET Deutsche WÖRTERbÜCHER UND GRAMMATIK, http://www.canoo.net. [10/ $11 / 2011]$.

CASARES, J., Diccionario ideológico de la lengua española. Barcelona: Gustavo Gili 1994.

Clave Diccionarios SM, http://clave.librosvivos.net. [30/09/2011].

CoP, M., "Collocations in the Bilingual Dictionary», en: HAUSMANN, F. J. (ed.), Wörterbücher. Ein Internationales Handbuch zur Lexikographie. Vol. 3. Berlín et al.: de Gruyter 1991, 2775-2779.

CORRIPIO, F., Gran diccionario de sinónimos, voces afines e incorrecciones. Barcelona: Ediciones B 1990.

CoSERIU, E., «Lexikalische Solidaritäten», Poetica 13 (1967), 293-303.

COSMAS II IDS, https://cosmas2.ids-mannheim.de. [25/10/2011].

CowIE A. P., «Information on Syntactic Constructions in the General Monolingual Dictionary», en: HAUSMANN, F. J. (ed.), Wörterbücher. Ein Internationales Handbuch zur Lexikographie. Vol. 1. Berlín et al.: de Gruyter 1989, 588-593.

DAVIES, M., Corpus del Español (100 million words, 1200s1900s). http://www.corpusdelespanol.org, 2002. [28/10/2011].

DORNSEIFF, F., Der deutsche Wortschatz nach Sachgruppen. Berlín: de Gruyter 1965.

DudEn, Deutsches Universalwörterbuch. Mannheim: Duden 2007.

DudEN, Das Stilwörterbuch neu. Mannheim: Duden 2001.

ENGEL, U. / SCHUMACHER, H., Kleines Valenzlexikon deutscher Verben. Tübingen: Gunter Narr 1978.

EnGEL, U., Deutsche Grammatik Neubearbeitung. Múnich: Iudicium 2004.

GECKELER, H., Semántica estructural y teoría del campo léxico. Madrid: Gredos 1994.

HARTMANN, R. R. K., "Contrastive Linguistics and Bilingual Lexicography», en: HAUSMANN, F. J. (ed.), Wörterbücher. Ein Internationales Handbuch zur Lexikographie. 3. Teilband. Berlín et al.: de Gruyter 1991, 2854-2859.

Hausmann, F. J., «Die Paradigmatik im zweisprachigen Wörterbuch», en: HaUsmanN, F. J. (ed.), Wörterbücher. Ein Internationales Handbuch zur Lexikographie. Vol. 3. Berlín et al.: de Gruyter 1991, 2794-2796.

HelBiG, G. / SchENKEL, W., Wörterbuch zur Valenz und Distribution deutscher Verben. Leipzig: VEB Bibliographisches Institut 1980. 
Model, B. A., «Sintagmática y diccionario bilingüe», Estudios Filológicos Alemanes 12 (2006), 247-256.

MOLINER, M., Diccionario de uso del español. Madrid: Gredos 2002.

OwID IDS, http://www.owid.de. [30/09/2011].

Peder Kromann, H. / RilBer, T. / Rosbach, P., "Grammatical Constructions in the Bilingual Dictionary», en: HAUSMANN F. J. (ed.), Wörterbücher. Ein Internationales Handbuch zur Lexikographie. 3. Teilband. Berlín et al.: de Gruyter 1991, 2770-2775.

PONS, Großwörterbuch für Experten und Universität. Barcelona: Klett 2003.

RADSZuWeit, S. / Spalier, M., Knaurs Lexikon der Synonyme. Der treffende Ausdruckdas passende Wort. Múnich: Knaur 1992.

Real ACADEmia EsPañola, Diccionario de la lengua. Vigésimo segunda edición, http://buscon.rae.es/draeI. [26/10/2011].

ReAl ACADEMIA EsPañola, Banco de datos (CREA) [en línea]. Corpus de referencia del español actual. http://www.rae.es. [26/10/2011].

REICHMANN, O., «Das onomasiologische Wörterbuch», en: HAUSMANN, F. J. (ed.), Wörterbücher. Ein Internationales Handbuch zur Lexikographie. 2. Teilband. Berlín et al.: de Gruyter 1990, 1057-1067.

SÁNCHEZ HERNÁNDEZ, P., "Análisis contrastivo alemán español de los verbos fragenantworten / lehren-lernen», Revista de Filología Alemana 18 (2010), 261-283.

SeCo, M. / ANDrÉS, O. / RAMos, G., Diccionario del español actual. Madrid: Aguilar 1999.

SECO, M., Diccionario de dudas y dificultades de la lengua española. Madrid: Espasa 2008.

SCHUmacher, H. / KubCZAK, J. / SCHMiDT, R. / De Ruiter, V., Valbu-Valenzwörterbuch deutscher Verben. Tübingen: Gunter Narr 2004.

WAHRIG, G., Deutsches Wörterbuch. Gütersloh: Bertelsmann 1997.

WeHrle, H. / EGGERS, H., Deutscher Wortschatz. Stuttgart: Klett 1961. 